\title{
On the divisor problem: Moments of $\Delta(x)$ over short intervals
}

by

\section{Werner Georg Nowak (Wien)}

1. Introduction. Let as usual $d(n)$ denote the number of (positive) divisors of the positive integer $n$. The classical Dirichlet divisor problem is concerned with estimates for the remainder term $\Delta(x)$ in the asymptotic formula

$$
D(x):=\sum_{n \leq x} d(n)=x \log x+(2 \gamma-1) x+\Delta(x),
$$

where $x$ is a large real variable and $\gamma$ is the Euler-Mascheroni constant. The sharpest upper bound published to date $\left({ }^{1}\right)$ is due to M. Huxley [5] and reads

$$
\Delta(x)=O\left(x^{23 / 73}(\log x)^{461 / 146}\right) .
$$

(For a survey of the history of the problem, the reader is referred to the monograph of E. Krätzel [8].) Concerning lower bounds, the best results $\operatorname{read}\left({ }^{2}\right)$

$$
\begin{array}{r}
\Delta(x)=\Omega_{+}\left(x^{1 / 4}(\log x)^{1 / 4}(\log \log x)^{(3+\log 4) / 4} \exp (-c \sqrt{\log \log \log x})\right) \\
(c>0),
\end{array}
$$

due to J. L. Hafner [3], and

$$
\Delta(x)=\Omega_{-}\left(x^{1 / 4} \exp \left(c^{\prime}(\log \log x)^{1 / 4}(\log \log \log x)^{-3 / 4}\right)\right) \quad\left(c^{\prime}>0\right),
$$

established by Corrádi and Kátai [2]. Most experts believe that in fact

$$
\Delta(x)=O\left(x^{1 / 4+\varepsilon}\right)
$$

for every $\varepsilon>0$. In favour of this conjecture, there is the classic mean-square

2000 Mathematics Subject Classification: Primary 11N37.

$\left({ }^{1}\right)$ Actually, M. Huxley has meanwhile further improved this estimate, essentially replacing the exponent $23 / 73=0.315068 \ldots$ by $131 / 416=0.3149038 \ldots$ The author is indebted to Professor Huxley for sending him a copy of his unpublished manuscript.

$\left({ }^{2}\right)$ Cf., e.g., [8] for the notations $O, \Omega, \ll$, and $\asymp$, used throughout the paper. 
asymptotics $\left({ }^{3}\right)$

$$
\int_{1}^{T}\left(\Delta\left(t^{2}\right)\right)^{2} d t=\frac{(\zeta(3 / 2))^{4}}{8 \pi^{2} \zeta(3)} T^{2}+O\left(T(\log T)^{4}\right) .
$$

This precise form of the error term was achieved by E. Preissmann [10], thus improving the estimate of K. C. Tong [12] who had the log-exponent 5 instead of 4. Only rather recently, K.-M. Tsang [13] found asymptotics for the third and fourth power moments $\left({ }^{4}\right)$. He proved that

$$
\int_{1}^{T}\left(\Delta\left(t^{2}\right)\right)^{3} d t=\frac{3 c_{3}}{40 \pi^{3}} T^{5 / 2}+O\left(T^{33 / 14+\varepsilon}\right)
$$

where

$$
\begin{aligned}
c_{3} & =\sum_{\substack{m, n, k=1 \\
\sqrt{m}+\sqrt{n}=\sqrt{k}}}^{\infty} \frac{d(m) d(n) d(k)}{(m n k)^{3 / 4}} \\
& =\sum_{q, r, s=1}^{\infty} \frac{|\mu(q)|}{q^{9 / 4}} d\left(r^{2} q\right) d\left(s^{2} q\right) d\left((r+s)^{2} q\right)(r s(r+s))^{-3 / 2},
\end{aligned}
$$

$\mu(\cdot)$ being the Möbius function, and

$$
\int_{1}^{T}\left(\Delta\left(t^{2}\right)\right)^{4} d t=\frac{c_{4}}{32 \pi^{4}} T^{3}+O\left(T^{67 / 23+\varepsilon}\right)
$$

with

$$
c_{4}=\sum_{\substack{m, n, k, l=1 \\ \sqrt{m}+\sqrt{n}=\sqrt{k}+\sqrt{l}}}^{\infty} \frac{d(m) d(n) d(k) d(l)}{(m n k l)^{3 / 4}} .
$$

We remark parenthetically that R. Heath-Brown [4] showed that the limits

$$
A_{m}=\lim _{T \rightarrow \infty}\left(\frac{1}{T^{1+m / 2}} \int_{1}^{T}\left(\Delta\left(t^{2}\right)\right)^{m} d t\right)
$$

exist for $m=1, \ldots, 9$. However, as Heath-Brown himself states, his argument is not able to decide if $A_{m}>0$ for $2 \leq m \leq 9$. In fact, $A_{1}=0$, by the

$\left({ }^{3}\right)$ In the context of integral means, we find it convenient to use $t=\sqrt{x}$ as a basic variable. If we imagine $D(x)$ as the number of lattice points under a hyperbola, then $t$ is a length parameter of the configuration. Changing the variable and integrating by parts, it is trivial to transform our asymptotics into the shape used in the literature cited, and vice versa.

$\left({ }^{4}\right)$ It is very interesting to read Tsang's interpretation of what his results, in particular the third moment asymptotics, reveal about the qualitative behaviour of $\Delta(x)$. 
very old result of Voronoï [14] that

$$
\int_{1}^{T}\left(\Delta\left(t^{2}\right)-1 / 4\right) d t=O\left(T^{1 / 2}\right) .
$$

2. Formulation of results. It is the aim of the present paper to point out that the "on average regular behaviour" of $\Delta(x)$, as displayed by the asymptotics cited, can be observed already if taking the respective means over much shorter intervals. In fact, it will be sufficient to average over $[T-\Lambda(T), T+\Lambda(T)]$, where for the quadratic case, $\Lambda(T)$ has to grow just faster than $(\log T)^{3}$, while for the third and fourth power moments, $\Lambda(T)$ must be at least of order $T^{\theta}$ with $\theta>1 / 2$.

Theorem 1. For $T$ a large real variable, suppose that $T \mapsto \Lambda=\Lambda(T)$ increases with $T$, satisfies $0<\Lambda(T) \leq \frac{1}{2} T$, and

$$
\lim _{T \rightarrow \infty} \frac{(\log T)^{3}}{\Lambda(T)}=0
$$

Then, as $T \rightarrow \infty$,

$$
\int_{T-\Lambda}^{T+\Lambda}\left(\Delta\left(t^{2}\right)\right)^{2} d t \sim \frac{1}{2 \pi^{2}} \frac{\zeta^{4}(3 / 2)}{\zeta(3)} \Lambda T .
$$

TheOREM 2. For $T, \Lambda(T)$ as above, suppose in addition that

$$
\lim _{T \rightarrow \infty} \frac{\Lambda(T)}{T}=: \lambda
$$

exists $\left({ }^{5}\right)$, and for some $\varepsilon_{0}>0$,

$$
\lim _{T \rightarrow \infty} \frac{T^{1 / 2+\varepsilon_{0}}}{\Lambda(T)}=0
$$

Then, as $T \rightarrow \infty$,

$$
\int_{T-\Lambda}^{T+\Lambda}\left(\Delta\left(t^{2}\right)\right)^{3} d t \sim \frac{3 c_{3} \beta(\lambda)}{16 \pi^{3}} \Lambda T^{3 / 2},
$$

with $\left({ }^{6}\right)$

$$
\beta(\lambda):= \begin{cases}2 & \text { for } \lambda=0, \\ \frac{2}{5 \lambda}\left((1+\lambda)^{5 / 2}-(1-\lambda)^{5 / 2}\right) & \text { else }\end{cases}
$$

$\left(^{5}\right)$ Of course, if $[T-\Lambda, T+\Lambda]$ is really a short interval, then $\lambda$ is 0 . We state the result for general $\lambda$ only to make possible an alternative approach to the asymptotics (1.6) and (1.7). See Remark 2 below.

$\left({ }^{6}\right)$ Obviously, $\lambda \mapsto \beta(\lambda)$ is continuous at $\lambda=0$. 
and

$$
\int_{T-\Lambda}^{T+\Lambda}\left(\Delta\left(t^{2}\right)\right)^{4} d t \sim \frac{3 c_{4}}{16 \pi^{4}}\left(1+\frac{1}{3} \lambda^{2}\right) \Lambda T^{2} .
$$

REMARKs. 1. Of course, (2.2) follows from (1.5), if (stronger than (2.1)) $\Lambda(T)$ grows faster than $(\log T)^{4}$. However, there is little hope to obtain the full strength of Theorem 1 via a corresponding improvement of (1.5). Similarly, (1.6), (1.7) imply (2.4), (2.6), resp., provided that $\Lambda(T)$ grows at least like $T^{\theta}, \theta>6 / 7$, resp. $\theta>21 / 23$. Compared to this, (2.3) is much less stringent.

2. If we take, in particular, $T=\frac{3}{4} X, \Lambda=\frac{1}{4} X$, the interval $[T-\Lambda$, $T+\Lambda]$ becomes $\left[\frac{1}{2} X, X\right]$, and $\lambda=1 / 3$. Using (2.4), resp. (2.6) for $X=$ $T, \frac{1}{2} T, \frac{1}{4} T, \ldots$ (with a new $T$ ), and summing up the geometric series arising, we reprove the asymptotics (1.6), resp. (1.7), though without obtaining an error term. However, our analysis is somewhat simpler than Tsang's.

3. Proof of Theorem 1. We start from the classic truncated Voronoï formula (see, e.g., Ivić [7, p. 86, (3.17)])

$$
\begin{array}{r}
\Delta\left(t^{2}\right)=\frac{\sqrt{t}}{\pi \sqrt{2}} \sum_{1 \leq n \leq N} \frac{d(n)}{n^{3 / 4}} \cos \left(4 \pi t \sqrt{n}-\frac{\pi}{4}\right)+O\left(N^{-1 / 2} t^{1+\varepsilon}\right) \\
(\varepsilon>0),
\end{array}
$$

where $N \ll t^{2}$ is any positive number. We apply this with $t \in[T-\Lambda(T)$, $T+\Lambda(T)], \Lambda=\Lambda(T)$ as in Theorem 1 , and $N=N(T) \ll T^{2}$ remaining at our disposal. Clearly we may infer that

$$
\Delta\left(t^{2}\right)=\frac{\sqrt{t}}{\pi \sqrt{2}} S(t)+O(\sqrt{t}|R(t)|)+O\left(N^{-1 / 2} T^{1+\varepsilon}\right),
$$

with

$$
S(t):=\sum_{1 \leq n \leq M} \frac{d(n)}{n^{3 / 4}} \cos \left(4 \pi t \sqrt{n}-\frac{\pi}{4}\right), \quad R(t):=\sum_{M<n \leq N} \frac{d(n)}{n^{3 / 4}} e(2 t \sqrt{n}),
$$

where $e(w)=e^{2 \pi i w}$ as usual, and $M$ is another large real parameter, independent $\left({ }^{7}\right)$ of $T$. We shall use further the simple fact that, for real functions $F, G$ defined on $I$ (with an obvious brief notation),

$$
\int_{I}(F+G)^{2}=\int_{I} F^{2}+O\left(\left(\int_{I} F^{2}\right)^{1 / 2}\left(\int_{I} G^{2}\right)^{1 / 2}\right)+O\left(\int_{I} G^{2}\right) .
$$

$\left({ }^{7}\right)$ Ultimately, $T$ will be sent to $+\infty$ while $M$ is kept fixed. We may thus imagine $T$ to be of order much larger than $M$. 
First we estimate the mean-square of $\sqrt{t}|R(t)|$, claiming that

$$
\int_{T-\Lambda}^{T+\Lambda}|\sqrt{t} R(t)|^{2} d t \ll T(\log T)^{3}+\Lambda T M^{-1 / 6+\varepsilon} \quad(\varepsilon>0) .
$$

To prove this, we employ a device which goes back to Huxley [6], and was developed further by the author [9]. It is based on the Fejér kernel

$$
\varphi(w):=\left(\frac{\sin (\pi w)}{\pi w}\right)^{2} .
$$

By Jordan's inequality, $\varphi(w) \geq 4 / \pi^{2}$ for $|w| \leq 1 / 2$, and the Fourier transform has the particularly simple shape

$$
\widehat{\varphi}(y)=\int_{\mathbb{R}} \varphi(w) e(w y) d w=\max (0,1-|y|) .
$$

Thus we conclude that

$$
\int_{T-\Lambda}^{T+\Lambda}|\sqrt{t} R(t)|^{2} d t \ll T \int_{T-\Lambda}^{T+\Lambda}|R(t)|^{2} d t=2 \Lambda T \int_{-1 / 2}^{1 / 2}|R(T+2 \Lambda w)|^{2} d w
$$$$
\leq \frac{1}{2} \pi^{2} \Lambda T \int_{\mathbb{R}}|R(T+2 \Lambda w)|^{2} \varphi(w) d w
$$$$
=\frac{1}{2} \pi^{2} \Lambda T \sum_{M<m, n \leq N} \frac{d(m) d(n)}{(m n)^{3 / 4}} e(2 T(\sqrt{n}-\sqrt{m})) \widehat{\varphi}(4 \Lambda(\sqrt{n}-\sqrt{m}))
$$$$
\ll \Lambda T \sum_{\substack{M<m, n \leq N \\|\sqrt{n}-\sqrt{m}|<1 /(4 \Lambda)}} \frac{d(m) d(n)}{(m n)^{3 / 4}} \ll \Lambda T \sum_{M<m \leq N} \frac{d(m)}{m^{3 / 2}} \sum_{\substack{n \in \mathbb{N}_{*} \\|\sqrt{n}-\sqrt{m}|<1 /(4 \Lambda)}} d(n)
$$

$$
\leq \Lambda T \sum_{M<m \leq N} \frac{d(m)}{m^{3 / 2}}\left(D\left(\left(\sqrt{m}+\frac{1}{4 \Lambda}\right)^{2}\right)-D\left(\left(\sqrt{m}-\frac{1}{4 \Lambda}\right)^{2}\right)\right) .
$$

By (1.1), with the easy bound $\Delta(x) \ll x^{1 / 3} \log x$ (see Krätzel [8, p. 198, Theorem 5.3]), it follows that, for arbitrary large reals $X_{1} \asymp X \asymp X_{2}$,

$$
D\left(X_{1}\right)-D\left(X_{2}\right) \ll\left|X_{1}-X_{2}\right| \log X+X^{1 / 3} \log X .
$$

Therefore, the last line in (3.5) is

$$
\begin{aligned}
& \ll \Lambda T \sum_{M<m \leq N} \frac{d(m)}{m^{3 / 2}}\left(\frac{\sqrt{m}}{\Lambda} \log m+m^{1 / 3} \log m\right) \\
& \ll T \sum_{1 \leq m \leq N} \frac{d(m)}{m} \log m+\Lambda T \sum_{m>M} m^{-7 / 6+\varepsilon} .
\end{aligned}
$$

But this is just $\ll$ the right hand side of (3.4), as asserted. 
We proceed to evaluate the mean-square of $(\sqrt{t} /(\pi \sqrt{2})) S(t)$. Let $S(t)^{2}=$ $S_{1}(t)+S_{2}(t)$ with

$$
\begin{aligned}
S_{1}(t) & :=\sum_{1 \leq m \leq M} \frac{d(m)^{2}}{m^{3 / 2}} \cos ^{2}\left(4 \pi t \sqrt{m}-\frac{\pi}{4}\right), \\
S_{2}(t) & :=\sum_{\substack{1 \leq m, n \leq M \\
m \neq n}} \frac{d(m) d(n)}{(m n)^{3 / 4}} \cos \left(4 \pi t \sqrt{m}-\frac{\pi}{4}\right) \cos \left(4 \pi t \sqrt{n}-\frac{\pi}{4}\right) .
\end{aligned}
$$

Using the elementary facts that, for arbitrary positive reals $A, B$,

$$
\int_{T-\Lambda}^{T+\Lambda} t \cos \left(A t-\frac{\pi}{4}\right) \cos \left(B t-\frac{\pi}{4}\right) d t= \begin{cases}\Lambda T+O(T / A) & \text { if } A=B \\ O(T /|A-B|) & \text { if } A \neq B\end{cases}
$$

we obtain

$$
\begin{aligned}
\frac{1}{2 \pi^{2}} \int_{T-\Lambda}^{T+\Lambda} t S_{1}(t) d t & =\frac{1}{2 \pi^{2}} \sum_{1 \leq m \leq M} \frac{d(m)^{2}}{m^{3 / 2}}\left(\Lambda T+O\left(\frac{T}{\sqrt{m}}\right)\right) \\
& =\frac{1}{2 \pi^{2}} \frac{\zeta^{4}(3 / 2)}{\zeta(3)} \Lambda T+O\left(\Lambda T M^{-1 / 2+\varepsilon}\right)+O(T)
\end{aligned}
$$

and

$$
\int_{T-\Lambda}^{T+\Lambda} t S_{2}(t) d t \ll T \sum_{\substack{1 \leq m, n \leq M \\ m \neq n}} \frac{d(m) d(n)}{(m n)^{3 / 4}|\sqrt{m}-\sqrt{n}|} \ll B_{1}(M) T .
$$

Here and throughout, $B_{j}(M), j=1,2, \ldots$, denote positive numbers depending only on $M$. Thus, altogether,

$$
\begin{aligned}
\int_{T-\Lambda}^{T+\Lambda}\left(\frac{\sqrt{t}}{\pi \sqrt{2}} S(t)\right)^{2} d t= & \frac{1}{2 \pi^{2}} \frac{\zeta^{4}(3 / 2)}{\zeta(3)} \Lambda T \\
& +O\left(\Lambda T M^{-1 / 2+\varepsilon}\right)+O\left(B_{1}(M) T\right) .
\end{aligned}
$$

For the present context, we choose $N=N(T)=T^{2}$. Using (3.2), (3.4), and (3.6) in (3.3), we arrive at

$$
\begin{aligned}
& \int_{T-\Lambda}^{T+\Lambda}\left(\Delta\left(t^{2}\right)\right)^{2} d t=\frac{1}{2 \pi^{2}} \frac{\zeta^{4}(3 / 2)}{\zeta(3)} \Lambda T+O\left(\Lambda T M^{-1 / 2+\varepsilon}\right)+O\left(B_{1}(M) T\right) \\
& +O\left(\left((\Lambda T)^{1 / 2}+B_{2}(M) T^{1 / 2}\right)\left(T^{1 / 2}(\log T)^{3 / 2}+(\Lambda T)^{1 / 2} M^{-1 / 12+\varepsilon}+\Lambda^{1 / 2} T^{\varepsilon}\right)\right) \\
& +O\left(T(\log T)^{3}+\Lambda T M^{-1 / 6+\varepsilon}+\Lambda T^{2 \varepsilon}\right) .
\end{aligned}
$$


Hence, in view of our condition (2.1),

$$
\limsup _{T \rightarrow \infty}\left|\frac{1}{\Lambda T} \int_{T-\Lambda}^{T+\Lambda}\left(\Delta\left(t^{2}\right)\right)^{2} d t-\frac{1}{2 \pi^{2}} \frac{\zeta^{4}(3 / 2)}{\zeta(3)}\right| \ll M^{-1 / 12+\varepsilon} .
$$

Since $M$ can be chosen arbitrarily large, this completes the proof of Theorem 1.

\section{Proof of Theorem 2}

4.1. The fourth power moment. Proof of (2.6). Again we start from the decomposition (3.2), but with

$$
N=N(T)=T^{4 / 5} \Lambda^{2 / 5}
$$

$\Lambda=\Lambda(T)$ satisfying the conditions of Theorem 2. Instead of (3.3) we now use the fact that, for real functions $F, G$,

$$
\int_{I}(F+G)^{4}=\int_{I} F^{4}+O\left(\left(\int_{I} F^{4}\right)^{3 / 4}\left(\int_{I} G^{4}\right)^{1 / 4}\right)+O\left(\int_{I} G^{4}\right) .
$$

This is immediate by Hölder's inequality. Furthermore, we shall need the following technical tool.

Lemma. Let $0<\delta<1$, and y a large real variable. Then

$$
\Sigma(\delta, y):=\sum_{\substack{(k, l) \in \mathbb{N}_{*}^{2} \\|\sqrt{k}+\sqrt{l}-y|<\delta}}(k l)^{-3 / 4} \ll \delta+y^{-4 / 3},
$$

the implied constant being absolute.

Proof. By symmetry, it suffices to estimate the corresponding sum restricted to $k \leq l$. This implies that $k \leq \frac{1}{4}(y+\delta)^{2}$ and $l \asymp y^{2}$. We use a dyadic subdivision of the $k$-range, putting $K=2^{-j-2}(y+\delta)^{2}, j=1,2, \ldots$ Then

$$
\begin{gathered}
\Sigma(\delta, y) \ll \sum_{\substack{K=2^{-j-2}(y+\delta)^{2} \\
j=1,2, \ldots}} K^{-3 / 4} y^{-3 / 2} \#\left(\mathcal{B}_{K}\right), \\
\mathcal{B}_{K}:=\left\{(k, l) \in \mathbb{N}_{*}^{2}: K<k \leq 2 K,|\sqrt{k}+\sqrt{l}-y|<\delta\right\} .
\end{gathered}
$$

Writing $\psi(w)=w-[w]-1 / 2,[w]$ the greatest integer $\leq w$, we get

$$
\begin{aligned}
\#\left(\mathcal{B}_{K}\right) \leq & \sum_{K<k \leq 2 K}\left(\left[(y-\sqrt{k}+\delta)^{2}\right]-\left[(y-\sqrt{k}-\delta)^{2}\right]\right) \\
\ll & \sum_{K<k \leq 2 K}\left((y-\sqrt{k}+\delta)^{2}-(y-\sqrt{k}-\delta)^{2}\right) \\
& +\sum_{ \pm}\left|\sum_{K<k \leq 2 K} \psi\left((y-\sqrt{k} \pm \delta)^{2}\right)\right| .
\end{aligned}
$$


The first sum is of course $\ll K y \delta$. To bound the last sums, we note that

$$
\frac{d^{2}}{d u^{2}}\left((y-\sqrt{u} \pm \delta)^{2}\right)=\frac{1}{2}(y \pm \delta) u^{-3 / 2} \asymp K^{-3 / 2} y,
$$

and use the simplest van der Corput estimate (see, e.g., Krätzel [8, p. 32, Theorem 2.3]) to conclude that

$$
\#\left(\mathcal{B}_{K}\right) \ll K y \delta+\min \left(K, K^{1 / 2} y^{1 / 3}+K^{3 / 4} y^{-1 / 2}\right) .
$$

Using this in (4.3), we obtain

$$
\Sigma(\delta, y) \ll \sum_{\substack{K=2^{-j-2}(y+\delta)^{2} \\ j=1,2, \ldots}}\left(K^{1 / 4} y^{-1 / 2} \delta+\min \left(K^{1 / 4} y^{-3 / 2}, K^{-1 / 4} y^{-7 / 6}+y^{-2}\right)\right) .
$$

Summing up the geometric series involved, and observing that $K^{1 / 4} y^{-3 / 2}<$ $K^{-1 / 4} y^{-7 / 6}$ iff $K<y^{2 / 3}$, we establish the Lemma.

To start the proof of Theorem 2, we first estimate the fourth moment of $\sqrt{t}|R(t)|$. The same argument which lead to (3.5) now gives

$$
\begin{aligned}
\int_{T-\Lambda}^{T+\Lambda}|\sqrt{t} R(t)|^{4} d t & \ll T^{2} \int_{T-\Lambda}^{T+\Lambda}|R(t)|^{4} d t=2 \Lambda T^{2} \int_{-1 / 2}^{1 / 2}|R(T+2 \Lambda w)|^{4} d w \\
& \leq \frac{1}{2} \pi^{2} \Lambda T^{2} \int_{\mathbb{R}}|R(T+2 \Lambda w)|^{4} \varphi(w) d w \\
& \ll \Lambda T^{2} \sum_{M<m, n, k, l \leq N}^{\substack{M<\sqrt{l}|<1 /(4 \Lambda)\\
| \sqrt{m}+\sqrt{n}-\sqrt{k}-\sqrt{l})}} \\
& \ll \Lambda T^{2} \sum_{M<m, n \leq N}(m n)^{-3 / 4+\varepsilon} \Sigma(1 /(4 \Lambda), \sqrt{m}+\sqrt{n}) .
\end{aligned}
$$

By our Lemma, this is

$$
\begin{aligned}
& \ll \Lambda T^{2} \sum_{M<m, n \leq N}(m n)^{-3 / 4+\varepsilon}\left(\frac{1}{\Lambda}+(\sqrt{m}+\sqrt{n})^{-4 / 3}\right) \\
& \ll T^{2} \sum_{1 \leq m, n \leq N}(m n)^{-3 / 4+\varepsilon}+\Lambda T^{2} \sum_{m, n>M}(m n)^{-13 / 12+\varepsilon} .
\end{aligned}
$$

Altogether we get, recalling (4.1),

$$
\int_{T-\Lambda}^{T+\Lambda}|\sqrt{t} R(t)|^{4} d t \ll \Lambda^{1 / 5} T^{12 / 5+\varepsilon^{\prime}}+\Lambda T^{2} M^{-1 / 6+\varepsilon^{\prime}}
$$

for any $\varepsilon^{\prime}>0$. 
It remains to evaluate the fourth moment of $\frac{\sqrt{t}}{\pi \sqrt{2}} S(t)$. By the elementary formula

$$
\cos (a) \cos (b) \cos (c) \cos (d)=\frac{1}{8} \sum_{ \pm} \cos (a \pm b \pm c \pm d),
$$

it follows that

$$
S(t)^{4}=\frac{3}{8} S_{3}+\frac{3}{8} S_{4}(t)+\frac{1}{2} S_{5}(t)+\frac{1}{8} S_{6}(t),
$$

where

$$
\begin{aligned}
S_{3} & :=\sum_{\substack{1 \leq m, n, k, l \leq M \\
\sqrt{m}+\sqrt{n}=\sqrt{k}+\sqrt{l}}} \frac{d(m) d(n) d(k) d(l)}{(m n k l)^{3 / 4}}, \\
S_{4}(t) & :=\sum_{\substack{1 \leq m, n, k, l \leq M \\
\sqrt{m}+\sqrt{n} \neq \sqrt{k}+\sqrt{l}}} \frac{d(m) d(n) d(k) d(l)}{(m n k l)^{3 / 4}} \cos (4 \pi t(\sqrt{m}+\sqrt{n}-\sqrt{k}-\sqrt{l})), \\
S_{5}(t) & :=\sum_{1 \leq m, n, k, l \leq M} \frac{d(m) d(n) d(k) d(l)}{(m n k l)^{3 / 4}} \sin (4 \pi t(\sqrt{m}+\sqrt{n}+\sqrt{k}-\sqrt{l})), \\
S_{6}(t) & :=-\sum_{1 \leq m, n, k, l \leq M} \frac{d(m) d(n) d(k) d(l)}{(m n k l)^{3 / 4}} \cos (4 \pi t(\sqrt{m}+\sqrt{n}+\sqrt{k}+\sqrt{l})) .
\end{aligned}
$$

Tsang $[13,(3.6)]$ already proved that

$$
S_{3}=c_{4}+O\left(M^{-1 / 4+\varepsilon}\right)
$$

on the basis of Besicovitch's [1] result that the square roots of square-free numbers are linearly independent over the rationals. Consequently,

$$
\int_{T-\Lambda}^{T+\Lambda}\left(\frac{\sqrt{t}}{\pi \sqrt{2}}\right)^{4} \frac{3}{8} S_{3} d t=\frac{3}{16 \pi^{4}}\left(\Lambda T^{2}+\frac{1}{3} \Lambda^{3}\right) c_{4}+O\left(\Lambda T^{2} M^{-1 / 4+\varepsilon}\right) .
$$

Furthermore, by the second mean-value theorem,

$$
\begin{aligned}
\int_{T-\Lambda}^{T+\Lambda} t^{2} S_{4}(t) d t \ll T^{2} & \sum_{\substack{1 \leq m, n, k, l \leq M \\
\sqrt{m}+\sqrt{n} \neq \sqrt{k}+\sqrt{l}}} \frac{d(m) d(n) d(k) d(l)}{(m n k l)^{3 / 4}|\sqrt{m}+\sqrt{n}-\sqrt{k}-\sqrt{l}|} \\
\ll & B_{3}(M) T^{2}, \\
\int_{T-\Lambda}^{T+\Lambda} t^{2} S_{5}(t) d t= & \sum_{\substack{1 \leq m, n, k, l \leq M \\
\sqrt{m}+\sqrt{n}+\sqrt{k} \neq \sqrt{l}}} \frac{d(m) d(n) d(k) d(l)}{(m n k l)^{3 / 4}} \\
& \times \int_{T-\Lambda}^{T+\Lambda} t^{2} \sin (4 \pi t(\sqrt{m}+\sqrt{n}+\sqrt{k}-\sqrt{l})) d t
\end{aligned}
$$




$$
\begin{aligned}
& \ll T^{2} \sum_{\substack{1 \leq m, n, k, l \leq M \\
\sqrt{m}+\sqrt{n}+\sqrt{k} \neq \sqrt{l}}} \frac{d(m) d(n) d(k) d(l)}{(m n k l)^{3 / 4}|\sqrt{m}+\sqrt{n}+\sqrt{k}-\sqrt{l}|} \\
& \ll B_{4}(M) T^{2}, \\
\int_{T-\Lambda}^{T+\Lambda} t^{2} S_{6}(t) d t & \ll T^{2} \sum_{1 \leq m, n, k, l \leq M} \frac{d(m) d(n) d(k) d(l)}{(m n k l)^{3 / 4}|\sqrt{m}+\sqrt{n}+\sqrt{k}+\sqrt{l}|} \\
& \ll B_{5}(M) T^{2} .
\end{aligned}
$$

Thus, altogether,

$$
\begin{aligned}
\int_{T-\Lambda}^{T+\Lambda}\left(\frac{\sqrt{t}}{\pi \sqrt{2}} S(t)\right)^{4} d t= & \frac{3}{16 \pi^{4}}\left(\Lambda T^{2}+\frac{1}{3} \Lambda^{3}\right) c_{4} \\
& +O\left(\Lambda T^{2} M^{-1 / 4+\varepsilon}\right)+O\left(B_{6}(M) T^{2}\right)
\end{aligned}
$$

Combining (3.2), (4.1), (4.4), and (4.6), we get, in view of (4.2),

$$
\begin{aligned}
& \int_{T-\Lambda}^{T+\Lambda}\left(\Delta\left(t^{2}\right)\right)^{4} d t=\frac{3}{16 \pi^{4}}\left(\Lambda T^{2}+\frac{1}{3} \Lambda^{3}\right) c_{4}+O\left(\Lambda T^{2} M^{-1 / 4+\varepsilon}\right)+O\left(B_{6}(M) T^{2}\right) \\
& \quad+O\left(\left(\Lambda^{3 / 4} T^{3 / 2}+B_{7}(M) T^{3 / 2}\right)\left(\Lambda^{1 / 20} T^{3 / 5+\varepsilon^{\prime}}+\Lambda^{1 / 4} T^{1 / 2} M^{-1 / 24+\varepsilon^{\prime}}\right)\right) \\
& \quad+O\left(\Lambda^{1 / 5} T^{12 / 5+\varepsilon^{\prime}}+\Lambda T^{2} M^{-1 / 6+\varepsilon^{\prime}}\right) .
\end{aligned}
$$

Therefore, by condition (2.3),

$$
\limsup _{T \rightarrow \infty}\left|\frac{1}{\Lambda T^{2}} \int_{T-\Lambda}^{T+\Lambda}\left(\Delta\left(t^{2}\right)\right)^{4} d t-\frac{3}{16 \pi^{4}} c_{4}\left(1+\frac{1}{3}\left(\frac{\Lambda}{T}\right)^{2}\right)\right| \ll M^{-1 / 24+\varepsilon^{\prime}} .
$$

Since $M$ can be chosen arbitrarily large, this completes the proof of (2.6).

4.2. The third power moment. Proof of (2.4). Actually, it will be rather simple to deduce the result for the third power from the estimates on the second and fourth power means already established. Looking back at (3.2), we put

$$
H(t):=\frac{\sqrt{t}}{\pi \sqrt{2}} S(t), \quad R^{*}(t):=\Delta\left(t^{2}\right)-H(t),
$$

and define, for $j=2,3,4$,

$$
H_{j}:=\int_{T-\Lambda}^{T+\Lambda} H(t)^{j} d t, \quad R_{j}:=\int_{T-\Lambda}^{T+\Lambda}\left|R^{*}(t)\right|^{j} d t
$$

The choice (4.1) is still valid. Then it is immediate from (3.2) and (3.4), 
resp. (4.4), that

$$
\begin{aligned}
& R_{2} \ll T(\log T)^{3}+\Lambda T M^{-1 / 7}+\Lambda^{3 / 5} T^{6 / 5+2 \varepsilon}, \\
& R_{4} \ll \Lambda^{1 / 5} T^{12 / 5+\varepsilon^{\prime}}+\Lambda T^{2} M^{-1 / 7},
\end{aligned}
$$

with $\varepsilon, \varepsilon^{\prime}>0$ as small as we want. Furthermore, by (3.6), resp. (4.6),

$$
H_{2} \ll \Lambda T+B_{1}(M) T, \quad H_{4} \ll \Lambda T^{2}+B_{6}(M) T^{2} .
$$

Therefore, by (4.7) and Cauchy's inequality,

$$
\text { 4.8) } \begin{aligned}
& \int_{T-\Lambda}^{T+\Lambda}\left(\Delta\left(t^{2}\right)\right)^{3} d t-H_{3} \ll\left(H_{4} R_{2}\right)^{1 / 2}+\left(H_{2} R_{4}\right)^{1 / 2}+\left(R_{2} R_{4}\right)^{1 / 2} \\
\ll & \left(\Lambda^{1 / 2} T+B_{8}(M) T\right)\left(T^{1 / 2}(\log T)^{3 / 2}+\Lambda^{1 / 2} T^{1 / 2} M^{-1 / 14}+\Lambda^{3 / 10} T^{3 / 5+\varepsilon}\right) \\
+ & \left(\Lambda^{1 / 2} T^{1 / 2}+B_{9}(M) T^{1 / 2}\right)\left(\Lambda^{1 / 10} T^{6 / 5+\varepsilon^{\prime}}+\Lambda^{1 / 2} T M^{-1 / 14}\right) \\
+ & \left(T^{1 / 2}(\log T)^{3 / 2}+\Lambda^{1 / 2} T^{1 / 2} M^{-1 / 14}+\Lambda^{3 / 10} T^{3 / 5+\varepsilon}\right) \\
\times & \left(\Lambda^{1 / 10} T^{6 / 5+\varepsilon^{\prime}}+\Lambda^{1 / 2} T M^{-1 / 14}\right) .
\end{aligned}
$$

It remains to evaluate $H_{3}$. To do this, we may closely follow Tsang [13], even with a bit of simplification. By the elementary identity

$$
\cos (a) \cos (b) \cos (c)=\frac{1}{4} \sum_{ \pm} \cos (a \pm b \pm c),
$$

it follows that

$$
S(t)^{3}=\frac{3}{4 \sqrt{2}} S_{7}+\frac{3}{4} S_{8}(t)+\frac{1}{4} S_{9}(t),
$$

with

$$
\begin{aligned}
S_{7} & :=\sum_{\substack{1 \leq m, n, k \leq M \\
\sqrt{m}+\sqrt{n}=\sqrt{k}}} \frac{d(m) d(n) d(k)}{(m n k)^{3 / 4}}, \\
S_{8}(t) & :=\sum_{\substack{1 \leq m, n, k \leq M \\
\sqrt{m}+\sqrt{n} \neq \sqrt{k}}} \frac{d(m) d(n) d(k)}{(m n k)^{3 / 4}} \cos \left(4 \pi t(\sqrt{m}+\sqrt{n}-\sqrt{k})-\frac{\pi}{4}\right), \\
S_{9}(t) & :=\sum_{1 \leq m, n, k \leq M} \frac{d(m) d(n) d(k)}{(m n k)^{3 / 4}} \cos \left(4 \pi t(\sqrt{m}+\sqrt{n}+\sqrt{k})-\frac{3 \pi}{4}\right) .
\end{aligned}
$$

By the analysis between formulae (2.11) and (2.12) of [13], Tsang has proved (again on the basis of Besicovitch's theorem [1]) that, in our notation,

$$
S_{7}=c_{3}+O\left(M^{-1+\varepsilon}\right) \text {. }
$$


Furthermore, by the second mean-value theorem,

$$
\begin{aligned}
\int_{T-\Lambda}^{T+\Lambda} t^{3 / 2} S_{8}(t) d t & \ll T^{3 / 2} \sum_{\substack{1 \leq m, n, k \leq M \\
\sqrt{m}+\sqrt{n} \neq \sqrt{k}}} \frac{d(m) d(n) d(k)}{(m n k)^{3 / 4}|\sqrt{m}+\sqrt{n}-\sqrt{k}|} \\
& \ll B_{10}(M) T^{3 / 2}, \\
\int_{T-\Lambda}^{T+\Lambda} t^{3 / 2} S_{9}(t) d t & \ll T^{3 / 2} \sum_{\substack{1 \leq m, n, k \leq M\\
}} \frac{d(m) d(n) d(k)}{(m n k)^{3 / 4}|\sqrt{m}+\sqrt{n}+\sqrt{k}|} \\
& \ll B_{11}(M) T^{3 / 2} .
\end{aligned}
$$

Therefore, altogether,

$$
\begin{aligned}
H_{3} & =\int_{T-\Lambda}^{T+\Lambda}\left(\frac{\sqrt{t}}{\pi \sqrt{2}} S(t)\right)^{3} d t \\
& =\frac{3 c_{3}}{16 \pi^{3}} I_{3}(T)+O\left(\Lambda T^{3 / 2} M^{-1+\varepsilon}\right)+O\left(B_{12}(M) T^{3 / 2}\right)
\end{aligned}
$$

where $I_{3}(T):=\int_{T-\Lambda}^{T+\Lambda} t^{3 / 2} d t$. Combining this with (4.8) and recalling condition (2.3), we conclude that

$$
\limsup _{T \rightarrow \infty}\left|\frac{1}{\Lambda T^{3 / 2}} \int_{T-\Lambda}^{T+\Lambda}\left(\Delta\left(t^{2}\right)\right)^{3} d t-\frac{3 c_{3}}{16 \pi^{3}} \frac{I_{3}(T)}{\Lambda T^{3 / 2}}\right| \ll M^{-1 / 14} .
$$

An easy calculation shows that

$$
\lim _{T \rightarrow \infty} \frac{I_{3}(T)}{\Lambda T^{3 / 2}}=\beta(\lambda),
$$

as defined by (2.5). Since in (4.9), $M$ is again arbitrary, the proof of (2.4) is thereby complete.

5. Concluding remark. When discussing the Dirichlet divisor problem, one immediately thinks of its "twin", the Gaussian circle problem. Let $r(n)$ denote the number of ways to write $n \geq 0$ as a sum of two squares; then the "lattice point discrepancy" $P(x)=\sum_{0 \leq n \leq x} r(n)-\pi x$ satisfies upper and lower estimates quite similar to those quoted for $\Delta(x)$ in the introduction. (See again Krätzel's monograph [8].) Concerning results analogous to those established above, an asymptotic formula for $\int_{T-\Lambda}^{T+\Lambda}\left(P\left(t^{2}\right)\right)^{2} d t$ is contained in the author's paper [9] where the case of a general planar domain with smooth boundary of nonzero curvature is discussed. Furthermore, the direct analogue of Theorem 2 can be deduced, for $P(x)$, by the very same 
proof, starting from the formula

$$
\begin{aligned}
& P\left(t^{2}\right)=-\frac{\sqrt{t}}{\pi} \sum_{1 \leq n \leq N} \frac{r(n)}{n^{3 / 4}} \cos \left(4 \pi t \sqrt{n}+\frac{\pi}{4}\right)+O\left(N^{-1 / 2} t^{1+\varepsilon}\right) \\
& \quad\left(\varepsilon>0, N \ll t^{2}\right) .
\end{aligned}
$$

This in turn follows on classic lines (cf. Ivić [7], or Titchmarsh [11]), if we replace the generating function $\zeta^{2}(s)$ of $d(n)$ by the Dedekind zeta-function $\zeta_{\mathbb{Q}(i)}(s)$.

\section{References}

[1] A. S. Besicovitch, On the linear independence of fractional powers of integers, J. London Math. Soc. 15 (1940), 3-6.

[2] K. Corrádi and I. Kátai, A comment on K. S. Gangadharan's paper "Two classical lattice point problems", Magyar Tud. Akad. Mat. Fiz. Oszt. Közl. 17 (1967), 89-97 (in Hungarian, with English summary).

[3] J. L. Hafner, New omega theorems for two classical lattice point problems, Invent. Math. 63 (1981), 181-186.

[4] D. R. Heath-Brown, The distribution and moments of the error term in the Dirichlet divisor problem, Acta Arith. 60 (1992), 389-415.

[5] M. N. Huxley, Exponential sums and lattice points, II, Proc. London Math. Soc. (3) 66 (1993), 279-301.

[6] -, The mean lattice point discrepancy, Proc. Edinburgh Math. Soc. 38 (1995), $523-531$.

[7] A. Ivić, The Riemann Zeta-Function, Wiley, New York, 1985.

[8] E. Krätzel, Lattice Points, Kluwer, Dordrecht, 1988.

[9] W. G. Nowak, On the mean lattice point discrepancy of a convex disc, Arch. Math. (Basel) 78 (2002), 241-248.

[10] E. Preissmann, Sur la moyenne quadratique du terme de reste du problème du cercle, C. R. Acad. Sci. Paris Sér. I Math. 306 (1988), 151-154.

[11] E. C. Titchmarsh, The Theory of the Riemann Zeta Function, Clarendon Press, Oxford, 1951.

[12] K. C. Tong, On divisor problems II, III, Acta Math. Sinica 6 (1956), 139-152, 515-541 (in Chinese, with English summary).

[13] K.-M. Tsang, Higher-power moments of $\Delta(x), E(t)$ and $P(x)$, Proc. London Math. Soc. (3) 65 (1992), 65-82.

[14] G. Voronoï, Sur une fonction transcendante et ses applications à la sommation de quelques séries, Ann. Sci. École Norm. Sup. (3) 21 (1904), 207-268, 459-534.

Institut für Mathematik und Angewandte Statistik

Universität für Bodenkultur

Peter Jordan-Straße 82

A-1190 Wien, Austria

E-mail: nowak@mail.boku.ac.at

Web: http://www.boku.ac.at/math/nth.html 